\section{Corts:} Sleep Medicine, University of Florida, Gainesville, Florida, USA

\section{Correspondence to} Dr Saminder Singh Kalra, University of Florida, Gainesville, FL 32610, USA;

dr_saminders@yahoo.com

Received 24 November 2019 Revised 31 December 2019

Accepted 13 January 2020

10 February 2020

\title{
Rapid development of bullous lung disease: a complication of electronic cigarette use
}

\author{
Saminder Singh Kalra, Faye Pais, Eloise Harman, Daniel Urbine
}

Published Online First

Electronic cigarettes (EC) use can result in acute lung injury with varying severity of the hypoxic respiratory failure. Spontaneous resolution can result with the cessation of EC use, although some patients with severe disease have required mechanical ventilation. We describe a previously healthy patient who presented with severe hypoxemic respiratory failure and developed a rapidly progressive bullous disease and bilateral pneumothoraces. The exponential increase in lung injury from EC use combined with limited mechanistic insights into the disease process highlights the importance of promptly recognising both the disease and its complications.

A 34-year-old man was admitted with a 1-week history of pleuritic chest pain, dyspnoea and subjective fevers. He had a 15-pack year history of smoking but quit 3 years prior when he transitioned to vaping with nicotine. Three months prior to admission he began using tetrahydrocannabinol via EC and by dabbing. The patient denied having any pulmonary symptoms prior to his current presentation and he had no previous chest imaging. On examination, he was tachypnoeic and tachycardic. An arterial blood gas on room air showed a $\mathrm{pH}$ of $7.46, \mathrm{PaCo}_{2}$ of $4.4 \mathrm{kPa}, \mathrm{PaO}_{2}$ of 8.5 $\mathrm{kPa}$ with $92 \% \mathrm{SaO}_{2}$. He was initially given supplemental oxygen via facemask. His oxygen requirements rapidly increased and he ultimately required 90\% high flow oxygen at 40 LPM to maintain an oxygen saturation of $90 \%$. He did not require invasive or non-invasive positive pressure ventilation. Initial chest radiograph showed bilateral parenchymal infiltrates with diffuse bilateral ground-glass opacities (GGOs) (figures 1 and 2 , left panel). The patient was started on antibiotics and corticosteroids. An evaluation of infectious, autoimmune and hypersensitivity pneumonitis aetiologies was unremarkable. On hospital day 12 he developed bilateral pneumothoraces requiring chest tube placement and video-assisted thoracoscopic surgery pleurodesis. CT imaging demonstrated large bilateral bullae absent on the CT at the time of admission (figures 1 and 2, right panel). As of November 2019, there were 2172 reported cases of acute, severe respiratory distress cases

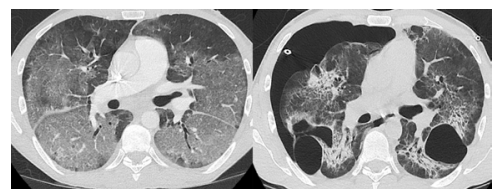
employer(s)) 2020. No commercial re-use. See rights and permissions. Published by BMJ.

To cite: Kalra SS, Pais F, Harman $\mathrm{E}$, et al. Thorax 2020;75:359.

Figure $1 \mathrm{CT}$ chest (transverse section). Left panel: bilateral parenchymal infiltrates with diffuse bilateral ground-glass opacities. Right panel: large bilateral bullae, ground glass opacities, bronchiectasis and right sided

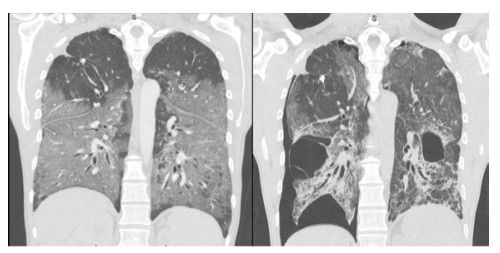

Figure 2 CT chest (coronal section). Left panel: bilateral parenchymal infiltrates with diffuse bilateral ground-glass opacities. Right panel: large bilateral bullae, ground glass opacities, bronchiectasis and right sided pneumothorax.

linked to vaping, spanning 49 states in the USA. These cases included a wide spectrum of diseases such as acute eosinophilic pneumonia, hypersensitivity pneumonitis, lipoid pneumonia, diffuse alveolar haemorrhage and acute respiratory distress syndrome. ${ }^{1}$

Radiographic findings typical of vaping related lung disease include GGOs, heterogeneous consolidation, crazy paving, micronodules alone or in combination. ${ }^{2}$ While bullous lung disease and pneumothoraces have been associated with chronic marijuana smoking described in the literature by one study reporting 57 cases, $^{3}$ the association and significance of bullae and emphysematous changes in patients who vape is unclear. Although Bonilla et al reported a case of recurrent pneumothoraces in a young male secondary to vaping, in that case, no residual lung disease was noted and the pneumothoraces resolved after chest tube placement. ${ }^{4}$ To our knowledge, this is the only case of vaping resulting in the rapid progression of bullous lung disease. Links between clinical outcomes, radiographic presentation, specific inciting agents and overall disease pathogenesis are yet to be defined in the heterogeneous vaping related lung diseases. In the interim, early recognition and management of complications is essential.

Competing interests None declared.

Patient consent for publication Obtained.

Provenance and peer review Not commissioned; externally peer reviewed.

\section{ORCID iD}

Daniel Urbine http://orcid.org/0000-0001-8022-6613

\section{REFERENCES}

1 Centers for Disease Control and Prevention. Outbreak of severe pulmonary disease associated with using e-cigarette products: investigation notice, 2019.

2 Landman ST, Dhaliwal I, Mackenzie CA, et al. Life-threatening bronchiolitis related to electronic cigarette use in a Canadian youth. CMAJ 2019;191:E1321-31. pneumothorax.
3 Ribeiro L, Ind PW. Marijuana and the lung: hysteria or cause for concern? Breathe 2018:14:196-205

4 Bonilla A, Blair AJ, Alamro SM, et al. Recurrent spontaneous pneumothoraces and vaping in an 18-year-old man: a case report and review of the literature. J Med Case Rep 2019;13:283. 